\title{
Umbilical endosalpingiosis: a case report
}

\author{
Theodossis S Papavramidis ${ }^{1 *}$, Konstantinos Sapalidis ${ }^{1}$, Nick Michalopoulos ${ }^{1}$, Georgia Karayannopoulou², \\ Angeliki Cheva², Spiros T Papavramidis ${ }^{1}$
}

\begin{abstract}
Introduction: Endosalpingiosis describes the ectopic growth of Fallopian tube epithelium. Pathology confirms the presence of a tube-like epithelium containing three types of cells: ciliated, columnar cells; non-ciliated, columnar secretory mucous cells; and intercalary cells.

We report the case of a woman with umbilical endosalpingiosis and examine the nature and characteristics of cutaneous endosalpingiosis by reviewing and combining the other four cases existing in the international literature.
\end{abstract}

Case presentation: A 50-year-old Caucasian, Greek woman presented with a pale brown nodule in her umbilicus. The nodule was asymptomatic, with no cyclical discomfort or variation in size. Her personal medical, surgical and gynecologic history was uneventful. An excision within healthy margins was performed under local anesthesia. A cystic formation measuring $2.7 \times 1.7 \times 1 \mathrm{~cm}$ was removed. Histological examination confirmed umbilical endosalpingiosis.

Conclusions: Umbilical endosalpingiosis is a very rare manifestation of the non-neoplasmatic disorders of the Müllerian system. It appears with cyclic symptoms of pain and swelling of the umbilicus, but not always. The disease is diagnosed using pathologic findings and surgical excision is the definitive treatment.

\section{Introduction}

Endosalpingiosis is a rare clinical entity that describes the ectopic growth of Fallopian tube epithelium [1]. Endosalpingiosis, endometriosis and endocervicosis constitute the triad of non-neoplastic disorders of the Müllerian system. These pathologies are found in isolation, but are more commonly found in association with one another $[2,3]$. The diagnosis of these pathologies is made histologically. In the case of endosalpingiosis, pathology confirms the presence of a tube-like epithelium containing three types of cells: ciliated, columnar cells; non-ciliated, columnar mucous secretor cells; and the so-called intercalary or peg cells $[4,5]$.

We report the case of an adult woman with umbilical endosalpingiosis and elucidate the nature and characteristics of cutaneous endosalpingiosis by reviewing and combining the four cases existing in the international literature.

\footnotetext{
* Correspondence: papavramidis@hotmail.com

'Department of Surgery, AHEPA University Hospital, Aristotle University of

Thessaloniki, Thessaloniki, Greece

Full list of author information is available at the end of the article
}

\section{Case presentation}

A 50-year-old Caucasian, Greek woman presented with a pale brown nodule in her umbilicus. The nodule was asymptomatic, with no cyclical discomfort or variation in size. Her personal medical, surgical and gynecologic history was uneventful. An excision within healthy margins was performed under local anesthesia. A cystic formation measuring $2.7 \times 1.7 \times 1 \mathrm{~cm}$ was removed. There were no signs of malignancy and no evidence of endometrial component in her surrounding tissue. Pathologic examination showed a unilocular cyst with papillary projections into the lumen (Figures 1 and 2). The cyst was surrounded by edematous fibrous tissue. The lining consisted of epithelium cells, both cuboidal and ciliate (Figure 3). Immunohistochemical staining showed positivity for keratins AE1/AE3 (Figure 4). Finally, a histological examination posed the diagnosis of cutaneous endosalpingiosis.

\section{Discussion}

The term endosalpingiosis was employed for the first time by Sampson et al. in 1930. Under that term, the author designated any unusual growth and invasion of
C Biomed Central

(ㄷ) 2010 Papavramidis et al; licensee BioMed Central Ltd. This is an Open Access article distributed under the terms of the Creative Commons Attribution License (http://creativecommons.org/licenses/by/2.0), which permits unrestricted use, distribution, and reproduction in any medium, provided the original work is properly cited. 


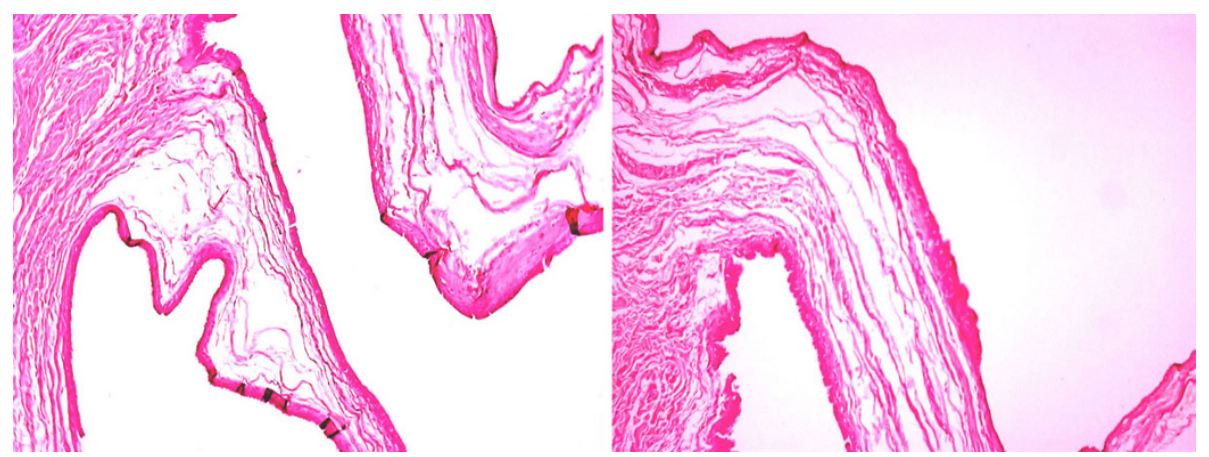

Figure 1 Unilocular cyst with papillary projections into the lumen (Hematoxylin and Eosin $\times 4$ )

tubal epithelium in tubal stumps, in subjects who had undergone previous salpingectomy or tubal sterilization [1]. The different theories for the pathogenesis of endosalpingiosis are similar to those for endometriosis, since those two entities, together with endocervicosis, constitute the non-neoplastic disorders of the Müllerian system. The different models can be traced back to two basic ideas. One group of theories is based on the fact that endometrial cells (or their precursors) are transported by various routes (transtubal, hematogenous, lymphogenous or by direct apposition) and implanted in the affected organ. The other group of theories suggests that Müllerian ectopias are the result of metaplastic processes in the target organ (coelomic metaplasia theory, secondary Müllerian system) or from scattered embryonic rest [6-8]. We believe that the first group of theories is inadequate when explaining the origin of endosalpingiosis in our case report, since she has a free gynecologic, obstetric and surgical history. In our case report, it is likely that the Müllerian ectopia resulted either from metaplastic processed or scattered embryonic rest.

Non-neoplastic glandular proliferation showing spontaneous Müllerian differentiation has been described in many sites including the vagina [9], uterine cervix [10], urinary bladder $[11,12]$, appendix [13], peritoneum
$[14,15]$, abdominal wall (inguinal channel, umbilicus) $[2,16]$, and the lymph nodes [17]. However, to the best of our knowledge, this is the first case of a patient with spontaneous endosalpingiosis presenting as a nodule on the abdominal wall.

The differential diagnosis of nodular umbilical lesions should include a wide range of diseases; such as hernia, keloid, abscess, lipoma, hematoma, sebaceous cyst, adenocarcinoma [primary of metastatic (Sister Joseph nodule)], melanoma, suture granuloma, pyogenic granuloma, desmoid tumor, sarcoma, lymphoma, endometriosis, and endosalpingiosis. Of course, the final diagnosis should be made by pathology [18].

In the international literature, this is the fifth case of umbilical endosalpingiosis. The other four cases refer to patients with previous medical history of gynecologic procedures $[5,19,20]$, while this is the first case of spontaneous appearance. These lesions appear as nodules of the umbilicus and are usually brownish in colour. The main symptoms (besides the esthetic) are pain and size fluctuation with menstruation. The treatment of choice is surgical excision. Our opinion is that excision has to be made under local anesthesia, in order to minimize morbidity and hospitalization. However, the patient has to be notified that in the case of a reappearance of

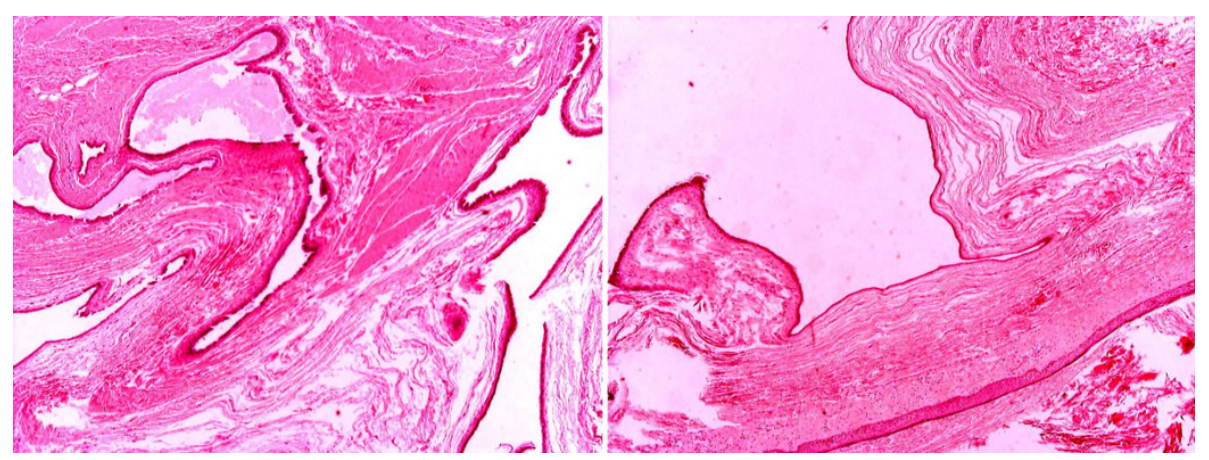

Figure 2 Unilocular cyst with papillary projections into the lumen (Hematoxylin and Eosin $\times 10$ ). 


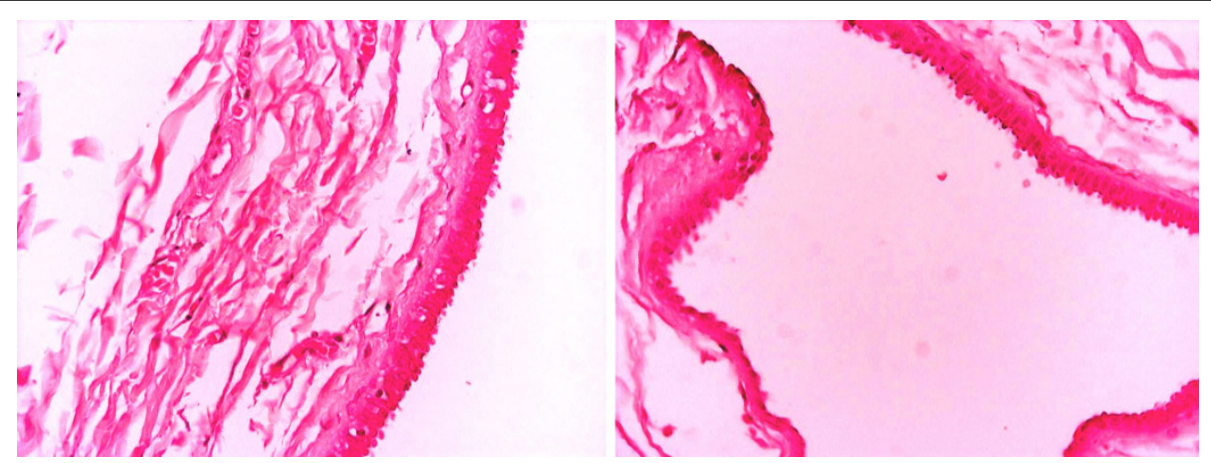

Figure 3 The lining of the cyst consisted of epithelium cells cuboidal and ciliate (Hematoxylin and Eosin $\times 40$ ).

abdominal pain (especially in the lower quadrant), a laparoscopy should be performed in order to exclude abdominal endometriosis.

\section{Conclusions}

Umbilical endosalpingiosis is a very rare manifestation of the non-neoplasmatic disorders of the Müllerian system. Normally, it appears with cyclic symptoms of pain and swelling of the umbilicus, but not always. The disease is diagnosed using pathologic findings and surgical excision is the definitive treatment.

\section{Consent}

Written informed consent was obtained from the patient for publication of this case report and any accompanying images. A copy of the written consent is available for review by the Editor-in-Chief of this journal.

\section{Competing interests}

The authors declare that they have no competing interests.

\section{Authors' contributions}

TSP analyzed and interpreted the patient data and drafted the manuscript. KS received the patient in our out-patient department and was the principal surgeon. NM received the patient in our out-patient department, was the auxillary surgeon and drafted the manuscript. GK performed the pathological examination and was a major contributor in writing the manuscript. AC performed the pathological examination. STP was responsible for the overall treatment of the patient and corrected the manuscript. All authors read and approved the final manuscript.

\section{Author details}

1 Department of Surgery, AHEPA University Hospital, Aristotle University of Thessaloniki, Thessaloniki, Greece. ${ }^{2}$ Department of Pathology, AHEPA University Hospital, Aristotle University of Thessaloniki, Thessaloniki, Greece.

Received: 23 October 2009 Accepted: 24 August 2010

Published: 24 August 2010

\section{References}

1. Sampson JA: Postsalpingectomy endometriosis (endosalpingiosis). Am J Obstet Gynecol 1930, 20:443-480.

2. Apostolidis S, Michalopoulos A, Papavramidis TS, Papadopoulos VN, Paramythiotis D, Harlaftis N: Inguinal endometriosis: three cases and literature review. South Med J 2009, 102:206-207.

3. Edmondson JD, Vogeley KJ, Howell JD, Koontz WW, Koo HP, Amaker B: Endosalpingiosis of bladder. J Urol 2002, 167:1401-1402.

4. Butterworth S, Stewart M, Clark JV: Heterotopic ciliated epithelium Müllerian origin? Lancet 1970, 1:1400-1401.

5. Redondo $\mathrm{P}$, Idoate $\mathrm{M}$, Corella C: Cutaneous umbilical endosalpingiosis with severe abdominal pain. J Eur Acad Dermatol Venereol 2001, 15:179-180.

6. Sinkre $\mathrm{P}$, Hoang MP, Albores-Saavedra J: Mullerianosis of inguinal lymph nodes: report of a case. Int J Gynecol Pathol 2002, 21:60-64.

7. Onybeke W, Brescia R, Eng K, Quagliarello J: Symptomatic endosalpingiosis in a postmenopausal woman. Am J Obstet Gynecol 1987, 156:924-926.

8. Keltz MD, Kliman HJ, Arici AM, Olive DL: Endosalpingiosis found at laparoscopy for chronic pelvic pain. Fertil Steril 1995, 64:482-485.

9. Martinka M, Allaire C, Clement PB: Endocervicosis presenting as a painful vaginal mass: a case report. Int J Gynecol Pathol 1999, 18:274-276.

10. Young $\mathrm{RH}$, Clement $\mathrm{PB}$ : Endocervicosis involving the uterine cervix: a report of four cases of a benign process that may be confused with deeply invasive endocervical adenocarcinoma. Int J Gynecol Pathol 2000 19:322-328.

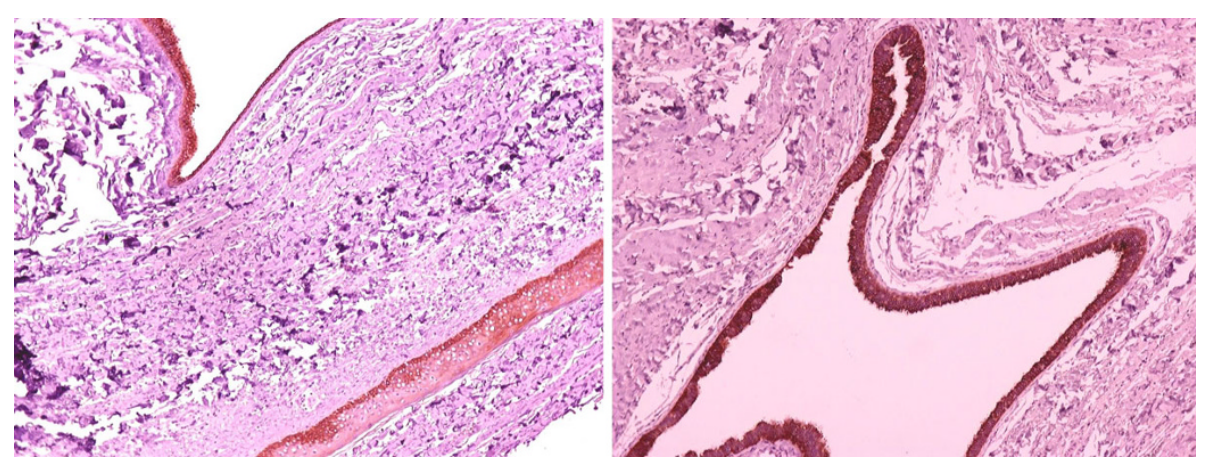

Figure 4 Immunohistochemical staining shows positivity for keratins AE1/AE3 (Keratins AE1/AE3 ×40). 
11. Nazeer T, Ro JY, Tornos C, Ordonez NG, Ayala AG: Endocervical type glands in urinary bladder: a clinicopathologic study of six cases. Hum Pathol 1996, 27:816-820.

12. Clement PB, Young RH: Endocervicosis of the urinary bladder. a report of six cases of a benign müllerian lesion that may mimic adenocarcinoma. Am J Surg Pathol 1992, 16:533-542.

13. Cajigas A, Axiotis CA: Endosalpingiosis of the vermiform appendix. Int $J$ Gynecol Pathol 1990, 9:291-295.

14. Lauchlan SC: The secondary müllerian system. Obstet Gynecol Surv 1972, 27:133-146.

15. Zinsser KR, Wheeler JE: Endosalpingiosis in the omentum: a study of autopsy and surgical material. Am J Surg Pathol 1982, 6:109-117.

16. Horton JD, Dezee KJ, Ahnfeldt EP, Wagner M: Abdominal wall endometriosis: a surgeon's perspective and review of 445 cases. Am J Surg 2008, 196:207-212.

17. Ferguson BR, Bennington JL, Haber SL: Histochemistry of mucosubstances and histology of mixed müllerian pelvic lymph node glandular inclusions. Evidence of histogenesis by müllerian metaplasia of coelomic epithelium. Obstet Gynecol 1969, 33:617-625.

18. Papavramidis TS, Sapalidis K, Michalopoulos N, Karayanopoulou G, Raptou G, Tzioufa V, Kesisoglou I, Papavramidis ST: Spontaneous abdominal wall endometriosis: a case report. Acta Chir Belg 2009, 109:778-781.

19. Doré N, Landry M, Cadotte M, Schürch W: Cutaneous endosalpingiosis. Arch Dermatol 1980, 116:909-912.

20. Perera GK, Watson KM, Salisbury J, Du Vivier AW: Two cases of cutaneous umbilical endosalpingiosis. Br J Dermatol 2004, 151:924-925.

doi:10.1186/1752-1947-4-287

Cite this article as: Papavramidis et al.: Umbilical endosalpingiosis: a case report. Journal of Medical Case Reports 2010 4:287.

\section{Submit your next manuscript to BioMed Central and take full advantage of:}

- Convenient online submission

- Thorough peer review

- No space constraints or color figure charges

- Immediate publication on acceptance

- Inclusion in PubMed, CAS, Scopus and Google Scholar

- Research which is freely available for redistribution

Submit your manuscript at www.biomedcentral.com/submit 